Abstract

\title{
Investigation of the Effect of Paclitaxel and Pycnogenol on Mitochondrial Dynamics in Breast Cancer Therapy ${ }^{\dagger}$
}

\author{
Suna Sayğı1ı 1,2,* , Hülya Birinci ${ }^{1}$, Büşra Şen ${ }^{1}$, Mustafa Öztatlıcı ${ }^{1}$, Sevinç İnan ${ }^{3}$ and \\ Kemal Özbilgin ${ }^{1}$ \\ 1 Department of Histology and Embryology, Faculty of Medicine, Manisa Celal Bayar University, \\ Manisa 45140, Turkey \\ 2 Department of Histology and Embryology, Faculty of Medicine, Dumlupınar University, \\ Kütahya 43100, Turkey \\ 3 Department of Histology and Embryology, Faculty of Medicine, Izmir Economy University, \\ Izmir 35330, Turkey \\ * Correspondence: suna_saygili@hotmail.com; Tel.: +90-553-6082149 \\ + Presented at the 2nd International Conference on Natural Products for Cancer Prevention and Therapy, \\ Kayseri, Turkey, 8-11 November 2017.
}

Published: 16 November 2017

\begin{abstract}
The aim of this study was to investigate the effects of microtubule organization inhibitor Paclitaxel and natural standardized flavonoid extract from the bark of French maritime pine (pycnogenol) on mitochondrial dynamics in breast cancer cell lines which have non-metastatic (67NR) and high metastatic (4T1) potential. 67NR and 4T1 breast cancer lines were cultured in DMEM-F12 medium and passaged every 2-3 days. Experimental groups is control group, paclitaxel group and pycnogenol group. We used $0.5 \mu \mathrm{M}$ for Paclitaxel and $20 \mu \mathrm{g}$ for pycnogenol and cells incubated $24 \mathrm{~h}$. Mfn-1 antibody for mitochondrial fusion, Drp-1 antibody for mitochondrial fission, Pink1 antibody for mitophagy were evaluated using indirect immunohistochemistry technique. The distributions of immunohistochemical intensities of primary antibodies were graded semiquantitatively. Scores of staining intensities were graded as mild, moderate, strong and very strong statistics were comparatively evaluated by using H-score. It was found that the usage of paclitaxel and pycnogenol were helpful in terms of cancer therapy. Immunohistochemical studies showed that Mfn-1, Drp-1 and Pink1 expressions significantly changed experimental groups for mitochondrial dynamics. Therefore, our study revealed that mitochondrial dynamics may be a potential target to improve the antineoplastic activity of paclitaxel and pycnogenol in breast cancer in the future.
\end{abstract}

Keywords: mitochondria; cancer; paclitaxel; pycnogenol

Conflicts of Interest: The authors declare no conflict of interest.

(C) 2017 by the authors. Licensee MDPI, Basel, Switzerland. This article is an open access article distributed under the terms and conditions of the Creative Commons Attribution (CC BY) license (http://creativecommons.org/licenses/by/4.0/). 